\title{
中国龙虾微卫星标记的篮选及遗传多样性分析
}

\author{
刘楚吾，黎锦明，刘丽，郭昱嵩 \\ 广东海洋大学水产学院, 南海水产经济动物增养殖广东普通高校重点实验室, 湛江 524025
}

摘要：文章以 $\mathrm{M}_{13}$ 通用引物和重复序列 $(\mathrm{CT})_{15} 、(\mathrm{AT})_{15}$ 引物，利用 $\mathrm{PCR}$ 法对中国龙虾(Panulirus stimpsoni Hoehuis) 部分基因组 DNA 文库进行篮选。共获得 78 个微卫星序列, 分别分布于 55 个阳性重组克隆中, 其中完美型 (perfect)共 50 个, 占 64\%; 非完美型(imperfect) 3 个，占 3.8\%; 混合完美型(compound perfect) 6 个，占 7.7\%; 混 合非完美型(compound imperfect) 19 个，占 $24.5 \%$ 。根据微卫星序列，设计并䇻选出 15 对微卫星多态性引物，对 中国龙虾的群体进行了遗传多样性分析。获得 3 12 个等位基因, 等位基因大小在 78 425 bp 之间, 基本符合引 物设计的理论长度。期望杂合度范围为 $0.48 \sim 0.87$, 平均值为 0.71 , 表明中国龙虾基因组微卫星具有较高的杂合 度与遗传多样性。15 个微卫星位点的 PIC 值从 0.44 到 0.84 , 平均值为 0.60 , 说明这些微卫星位点在中国龙虾基 因组中包含丰富的遗传信息，合适用于中国龙虾的各种分子标记及遗传学分析和应用。

关键词: 中国龙虾; 基因组文库; 微卫星标记; 遗传多样性；引物節选

\section{Screening and genetic diversity analysis of microsatellite markers in Chinese lobster(Panulirus stimpsoni)}

\author{
LIU Chu-Wu, LI Jin-Ming, LIU Li, GUO Yu-Song \\ Fisheries College, Key Laboratory of Aquaculture in South China Sea for Aquatic Economic Animal of Guangdong Higher Education \\ Institutes, Guangdong Ocean University, Zhanjiang 524025, China
}

\begin{abstract}
With the construction of a library of partial fractionated genomic DNA of Panulirus stimpsoni Hoehuis, the microsatellite sequences of $P$. stimpsoni were screened by PCR technique. Then, the genetic diversity was analyzed with the microsatellite markers. Seventy-eight microsatellite sequences in 55 positive recombinant clones were obtained by PCR technique with primers of M13+/- and $(\mathrm{CT})_{15}$, and $(\mathrm{AT})_{15}$. Among these microsatellite sequences, the numbers of perfect, imperfect, compound perfect, and compound imperfect sequences were 50 (64\%), 3 (3.8\%), 5 (7.7\%), and 19 (24.5\%), respectively. To analyze genomic DNA diversity of $P$. stimpsoni, 15 pairs of primers were designed from the microsatellite flanking sequences. In these microsatellite loci, the alleles numbers ranged from 3 to 12; and the sizes of these alleles ranged from 78 to $425 \mathrm{bp}$, which are in accordance with their predicted size range. The expected heterozygosity $\left(H_{\mathrm{e}}\right)$ and the polymorphism information content (PIC) ranged from 0.48 to 0.87 and 0.44 to 0.84 with the average values of 0.71 and 0.60 , respectively. These results showed that these microsatellite loci were suitable for $P$. stimpsoni molecule markers and genetic analysis because of their richness in genetic information.
\end{abstract}

Keywords: Panulirus stimpsoni; genomic library; microsatellite markers; genetic diversity; primer screen

微卫星 (Microsatellite), 又称简单序列重复 (Simple sequence repeat, SSR)、短串联重复(Short tandem repeat, STR), 是以少数几个核苷酸 (一般 $2 \sim 4$ 个)为重复单位的串联重复 DNA 序列 ${ }^{[1]}$ 。微卫 
星位点广泛分布于真核生物的基因组中, 具有高度 多态性和丰富的信息含量, 且符合孟德尔遗传和具 有共显性 ${ }^{[2]}$ 。微卫星标记的这些特点使它能够用于 研究等位基因, 区分二倍体(或多倍体)的纯合体或 杂合体。另外, 微卫星的特异性引物扩增具有良好 的重复性和保真性, 方便各实验室间的交流。目前, 微卫星标记已被广泛应用到基因连锁与遗传图谱构 建 $^{[3]}$ 、遗传多样性研究 ${ }^{[4 \sim 6]}$ 、谱系和发育研究 ${ }^{[7,8]}$ 、疾 病检测 ${ }^{[9]}$ 以及品种鉴定、亲本分析与个体、纯系检 验上 ${ }^{[10,11]}$ 。

中国龙虾(Panulirus stimpsoni Hoehuis)隶属甲壳 纲 (Crustacea)、十足目 (Decapoda)、爬行亚目 (Reptantia)、龙虾科(Palinuridae)、龙虾属(Panulirus) ${ }^{[12]}$ 。 我国龙虾资源丰富，在温带、亚热带、热带海区都 有分布, 是十分重要的渔业资源之一。我国对中国 海域龙虾类的研究早在上世纪的 60 年代就已经开 始 ${ }^{[13]}$, 主要的研究对象为我国海域产量最大的中国 龙虾, 70 年代纪成林等 ${ }^{[12]}$ 对其作了形态学和生态学 上的初步研究。到目前为止, 虽然对我国海域龙虾类 的研究已经逐步展开, 在个体发生及亲虾培育、人工 繁殖、早期叶状幼体的培育、盐度对中国龙虾的存 活及生长的影响等方面都有研究, 但对我国海域龙 虾类分子生物学方面的研究尚未见报道。

国外学者对龙虾类的配子发生 ${ }^{[14,15]}$ 、胚胎发

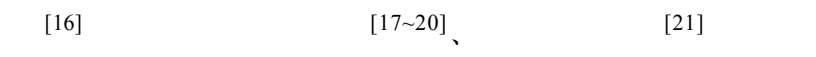
进行了大量研究, 但对龙虾类分子生物学方面的研 究不多。Garcia 等 ${ }^{[22]}$ 运用 mtDNA 的 RFLP 技术研究 了美国加利福尼亚东部沿海的断沟龙虾(Panulirus interruptus Randall)的遗传多样性。Chow 等 ${ }^{[23]}$ 则用 Alu I 和 Taq I 两种限制性内切酶对太平洋西北部的 日本海域龙虾种类叶状幼虫进行了 RFLP 分析。 Diniz 等 ${ }^{[24]}$ 建立了美国加利福尼亚北部沿海眼斑龙 虾(Panulirus argus Latreille)的部分基因组文库，并 从中篮选到了 9 对可用于眼斑龙虾群体分子标记的 微卫星引物。Hodgins 等 ${ }^{[25]}$ 分析了 8 个来自美国龙 虾(Homarus americanus Milne-Edwards)的微卫星序 列, 并把其中的 4 个用于挪威龙虾的研究。Groth 等 ${ }^{[26]}$ 则用两种不同的方法获得了 9 个西方岩龙虾 (Panulirus cygnus George)的微卫星序列, 用以研究 其种群结构和交配系统。

本文建立了中国龙虾部分基因组文库，采用
PCR 法篮选其微卫星序列, 并利用这些微卫星标记 对中国龙虾种群资源进行遗传多样性分析, 为今后 中国龙虾的种质资源保护和鉴定及中国龙虾资源进 一步开发利用等提供理论依据和指导。

\section{1 材料和方法}

\section{1 实验材料}

中国龙虾从广东湛江市霞山水产批发市场购得。

1.2 方法

\subsection{1 中国龙虾基因组 DNA 的提取}

参照卢圣栋等 ${ }^{[27]}$ 的方法, 从中国龙虾尾部肌肉 中提取基因组 DNA, 溶解于 TE 溶液中, 利用紫外 分光光度计检测浓度及纯度, $4^{\circ} \mathrm{C}$ 保存。

\subsection{2 中国龙虾基因组 DNA 的酶切}

使用限制性内切酶Sau 3A I 酶切中国龙虾基因 组 DNA，1\%琼脂糖凝胶电泳分离酶切产物，选取片 段大小在 200 1500 bp 之间的酶切产物进行回收、 纯化，用于克隆连接。

\subsection{3 中国龙虾部分基因组文库的构建}

将经限制性内切酶 Sau 3A I 酶切后的中国龙虾 基因组 DNA 片段与经 BamH I 酶切并去磷酸化的 pUC118 质粒载体进行重组, 重组体转入大肠杆菌 DH5 $\alpha$ 后利用菌落的蓝白斑指示篮选阳性克隆, 从 而构建中国龙虾小片段部分基因组 DNA 文库。

\subsection{4 用 PCR 法篎选含有微卫星的阳性克隆}

以 $\mathrm{M} 13$ 通用引物和自行设计合成的重复序列 $(\mathrm{CT})_{15} 、(\mathrm{AT})_{15}$ 探针引物分别对阳性克隆菌进行 PCR 扩增, PCR 反应总体积 $25 \mu \mathrm{L}$, 含 $1 \times \mathrm{PCR}$ Buffer $2 \mu \mathrm{L}$, $\mathrm{MgCl}_{2}(1.5 \mathrm{mmol} / \mathrm{L}) 2 \mu \mathrm{L}, \operatorname{dNTPs}(0.2 \mathrm{mmol} / \mathrm{L}) 2 \mu \mathrm{L}$, 引物各 $1 \mu \mathrm{mol} / \mathrm{L}, T a q$ 聚合酶 $2 \mathrm{U}$, 基因组 DNA 约 $30 \mathrm{ng}$, 加无菌水至总体积 $25 \mu \mathrm{L}$ 。1\%琼脂糖电泳分 离产物, 将 $\mathrm{M} 13+/(\mathrm{CT})_{15} 、 \mathrm{M} 13-/(\mathrm{CT})_{15} 、 \mathrm{M} 13+/(\mathrm{AT})_{15}$ 或 M13-/(AT) 15 具条带且条带比 M13+/-扩增产物小 的阳性克隆, 进行二次篮选, 若再次出现同样大小 的条带，可认为阳性克隆中可能含有微卫星序列。

\subsection{5阳性克隆的序列测定及结果分析}

对 PCR 结果为阳性的克隆委托上海生物工程技 
术有限公司测序, 利用 SSR Hunter 软件进行 SSR 重 复序列的寻找, 确定重复次数以及两端侧翼序列。

\subsection{6 微卫星引物的设计}

根据中国龙虾微卫星核心序列的侧翼序列，选 取符合引物设计的微卫星核心序列, 应用引物设计 软件 Primer 5.0 进行微卫星引物的设计。引物长度为 $20 \mathrm{bp}$ 左右, $(\mathrm{G}+\mathrm{C}) \%$ 为 $40 \% \sim 60 \%$, $\mathrm{Tm}$ 值为 $55 \sim 60^{\circ} \mathrm{C}$, 产物长度 100 400 bp, 由上海生工生物工程技术服 务有限公司合成。

\subsubsection{PCR 扩增与电泳检测}

利用合成的微卫星引物对 3 个中国龙虾个体的 基因组 DNA 混合样品进行 PCR 扩增分析。PCR 反 应体积为 $15 \mu \mathrm{L}$, 其中含 $10 \times$ Buffer $1.5 \mu \mathrm{L}$, dNTPs $(2 \mathrm{mmol} / \mathrm{L}) 1.5 \mu \mathrm{L}, \mathrm{Mg}^{2+}(25 \mathrm{mmol} / \mathrm{L}) 0.6 \mu \mathrm{L}, 30 \mu \mathrm{mol} / \mathrm{L}$ 的 $5^{\prime}$ 和 $3^{\prime}$ 端引物各 $1.5 \mu \mathrm{L}, \mathrm{Taq}$ 酶 $0.2 \mu \mathrm{L}$, 模板 DNA $0.8 \mu \mathrm{L}$, 无菌双蒸水 $7.4 \mu \mathrm{L}$ 。扩增程序: $94^{\circ} \mathrm{C}$ 预变性 $5 \mathrm{~min} ; 94^{\circ} \mathrm{C}$ 变性 $42 \mathrm{~s}, 45 \sim 60^{\circ} \mathrm{C}$ 复性 $50 \mathrm{~s}, 72^{\circ} \mathrm{C}$ 延伸 $45 \mathrm{~s}, 30$ 个循环; 最后再 $72^{\circ} \mathrm{C}$ 延伸 $5 \mathrm{~min}$ 。用 $8 \%$ 聚丙 烯酰胺凝胶电泳银染检测。

\subsection{8 数据统计分析}

根据每个个体产生的条带位置确定基因型, 利 用 GENEPOP Version 3.4 $4^{[28]}$ 计算等位基因数、观测杂 合度 (Observed heterozygosity，Ho) 和期望杂合度 (Expected heterozygosity, $\mathrm{He}$ ), 并进行多态性信息含 量(Polymorphism information content, PIC)的计算和 哈代 - 温伯格平衡 (Hardy-Weinberg equilibrium, HWE)检测。

$$
H o=\frac{1}{n} \sum_{i=1}^{n}\left(1-\sum_{j=1}^{l i} P_{i j}\right)=\frac{1}{n} \sum \text { hoi } ; \quad H e=1-\sum_{i=1}^{m} p_{1}^{2}
$$

式中: $h o i=1-\sum_{j=1}^{l i} P_{i j}$ 为第 $i$ 个位点上实际杂合体比 率; $P_{i j}$ 为第 $i$ 个位点上第 $j$ 个纯合体的频率; $P_{i}$ 为该 座位第 $i$ 等位基因频率。

$$
P I C=1-\sum_{i=1}^{m} p_{i}^{2}-\sum_{i=1}^{m-1} \sum_{j=i+1}^{m} p_{i}^{2} p_{j}^{2} ; \overline{P I C}=\frac{1}{L} \sum_{i=1}^{m} P I C
$$

$\overline{P I C}$ ：位点平均多态信息含量; $L$ 为微卫星位 点数; $P_{i}$ 和 $P_{j}$ 分别为第 $i$ 和第 $j$ 个等位基因在群体 中的频率; $m$ 为等位基因数。

\section{2 结果与分析}

\subsection{PCR 法筛选微卫星阳性克隆}

用 M13+/-通用引物对阳性克隆菌进行 PCR 检 测，结果显示，插入片段的大小集中在 100 1000 bp 之间。

以 $\mathrm{M} 13+/-$ 通用引物和自行设计合成的重复序 列 $(\mathrm{CT})_{15} 、(\mathrm{AT})_{15}$ 探针引物分别对阳性克隆菌进行 PCR 扩增, 篮选微卫星序列。以 $\mathrm{M} 13+/(\mathrm{CT})_{15}$ 为引 物的 PCR 产物的电泳结果(图 1)为例, 第 5 泳道的阳 性克隆菌可能含有 $(\mathrm{CT})$ 类型的微卫星序列。

\section{2 篮选后阳性克隆的测序结果}

对经过 PCR 初步篮选得到的 57 个可能含有微 卫星的重组阳性克隆进行 DNA 测序，证实在 57 个 重组克隆中有 55 个克隆含有微卫星序列，共含有 78 个微卫星序列，用来设计引物的序列见表 1 。

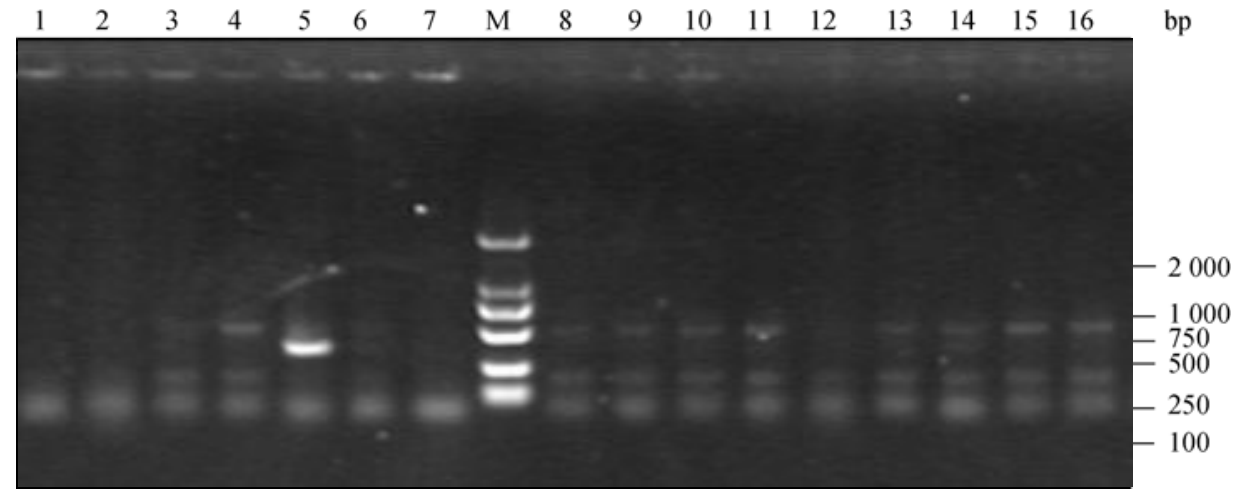

图 1 引物 $\mathrm{M} 13+/(\mathrm{CT})_{15}$ 对阳性克隆菌的 PCR 产物电泳图

M: DL2000 Markers; 1 16: 以 M13+/(CT) 15 为引物对阳性克隆进行 PCR 扩增, 其中 5 泳道对应的阳性克隆可能含有 $(\mathrm{CT})$ 类型微卫星序列, 其他 则无该类型微卫星序列。 
表 1 中国龙虾部分微卫星核心序列及其 $5^{\prime}$ 端与 3 端保守序列

\begin{tabular}{|c|c|c|c|c|c|}
\hline 克隆编号 & $5^{\prime}$ 旁侧序列 & 核心序列 & $3^{\prime}$ 旁侧序列 & 类别 & $\begin{array}{c}\text { GenBank } \\
\text { 登录号 }\end{array}$ \\
\hline Clone2 & TGTTGGGGAT & $(\mathrm{GA})_{4}(\mathrm{GTT}) \mathrm{GG}(\mathrm{GA})_{2} \mathrm{~T}(\mathrm{GA})(\mathrm{GT})(\mathrm{CA})(\mathrm{GTT})_{3}$ & CGCTGATGAT & 混合非完美型 & EU557281 \\
\hline Clone3 & ATCATCAGCG & $(\mathrm{AAC})_{3}(\mathrm{TG})(\mathrm{ACTC})_{2}(\mathrm{CCA})(\mathrm{A})(\mathrm{CT})_{3}$ & CATCCCCAAC & 混合非完美型 & EU557282 \\
\hline Clone6 & AATGATGATA & $(\mathrm{TAA})_{4}$ & TCGTACTGCT & 完美型 & EU557283 \\
\hline Clone6 & AAGCAGTACG & $(\mathrm{ATT})_{4}(\mathrm{AT})_{2}(\mathrm{CAT})_{2}$ & TGATCTGGAC & 非完美型 & \\
\hline Clone9 & GTGTAGCAAG & $\begin{array}{l}(\mathrm{CT})(\mathrm{TG})_{2}(\mathrm{CT})(\mathrm{GGA})_{3}(\mathrm{TG})_{2}(\mathrm{AGG})_{2} \\
(\mathrm{AC})(\mathrm{CT})(\mathrm{GT})(\mathrm{AGG})_{3}\end{array}$ & TATGGGCCAG & 混合非完美型 & EU557286 \\
\hline Clone9 & GATGGTATCG & $(\mathrm{GT})_{5}(\mathrm{GA})_{2}(\mathrm{GT})_{14}(\mathrm{TTG})_{2}$ & AGTGACCGGG & 混合完美型 & EU557287 \\
\hline Clone9 & CGACCACTCA & $(\mathrm{AC})_{16}(\mathrm{CT})(\mathrm{CA})_{5}$ & CCGATACCAT & 非完美型 & EU557288 \\
\hline Clone 10 & GCGGCATTAA & $(\mathrm{CG})_{3} \mathrm{GG}(\mathrm{TG})_{5}$ & TTACGTAGGA & 混合非完美型 & EU557289 \\
\hline Clone11 & GCATTCATCG & $(\mathrm{CA})_{4}(\mathrm{TA})(\mathrm{TG})_{3}$ & TCGTTAAAGG & 混合非完美型 & EU557290 \\
\hline Clone 18 & ACGTGGCTTG & $(\mathrm{CAT})_{2}(\mathrm{CG})(\mathrm{CA})_{4}$ & TATGTGTCGT & 混合非完美型 & EU557291 \\
\hline Clone22 & GCAAAGTGAA & $(\mathrm{ATT})_{3} \mathrm{~A}(\mathrm{AG})_{4}$ & AACTAAAAGA & 混合非完美型 & EU557292 \\
\hline Clone27 & CCACTAACGC & $(\mathrm{TA})_{4}(\mathrm{TG})_{3}(\mathrm{TA})_{3}$ & TTCCTATGAG & 混合完美型 & EU557293 \\
\hline Clone27 & TGTCCAAAAA & $(\mathrm{TG})_{3}(\mathrm{TA})_{17}$ & TGCCAGGTAC & 混合完美型 & EU557294 \\
\hline Clone27 & GGGCTAGGTC & $(\mathrm{AT})_{23} \mathrm{G}(\mathrm{TA})_{5} \mathrm{G}(\mathrm{AT})_{8}$ & СТСТTCTATG & 非完美型 & EU557295 \\
\hline Clone27 & ACTCATAGGA & (AT)3(AC)2(AT)5 & CGTTAGTGGG & 混合完美型 & EU557296 \\
\hline Clone 30 & AAGCGCGGCG & $(\mathrm{GGT})_{4}$ & TACGCGCAGC & 完美型 & EU557297 \\
\hline Clone44 & GCAAAGTGAA & $(\mathrm{ATT})_{3} \mathrm{~A}_{3}(\mathrm{AG})_{4}$ & AACTAAAAGA & 混合非完美型 & EU557298 \\
\hline Clone47 & GAACCCCTCA & $\begin{array}{l}(\mathrm{TC}) \mathrm{C}(\mathrm{TC})(\mathrm{CT}) 3(\mathrm{AC})(\mathrm{CT})(\mathrm{AC}) \\
(\mathrm{TA})(\mathrm{AC})_{3}\end{array}$ & ATGCCTCGCA & 混合非完美型 & EU557299 \\
\hline Clone47 & ATATGAGGCA & $(\mathrm{TG})_{2}(\mathrm{TA})(\mathrm{TG})_{3} \mathrm{G}(\mathrm{GA})(\mathrm{AG})_{3}$ & GGGAGTGGTT & 混合非完美型 & EU557300 \\
\hline Clone48 & TTTGCATTAT & $(\mathrm{TG})_{3}(\mathrm{TA})_{73}$ & TGTCCGATAT & 混合完美型 & EU595408 \\
\hline Clone 52 & & $(\mathrm{GGA})_{3}(\mathrm{TG})_{2}(\mathrm{AGG})_{2}(\mathrm{AC})_{2}(\mathrm{CT})(\mathrm{GC})(\mathrm{AGG})_{3}$ & TATGGGCCAG & 混合非完美型 & EU557301 \\
\hline Clone 53 & CCAGCTCAAT & $(\mathrm{GAT})_{2} \mathrm{~A}(\mathrm{TAA})_{4}$ & CCGAGTATCA & 混合非完美型 & EU557302 \\
\hline Clone 54 & GTAATGAGTA & $(\mathrm{TC})_{3}(\mathrm{AGAT}) \mathrm{TT}(\mathrm{AGAT})(\mathrm{AC})(\mathrm{TG})_{4}$ & ССТСАТТССС & 混合非完美型 & EU557303 \\
\hline Clone67 & CCCAACCTGC & $(\mathrm{GT})_{3}(\mathrm{GC})(\mathrm{GAA})(\mathrm{AC})_{4}$ & TCGTTAAAGG & 混合非完美型 & EU557304 \\
\hline Clone68 & GCATTCATCG & $(\mathrm{CA})_{4}(\mathrm{TA})(\mathrm{TG})_{3}$ & AGTTAACACA & 混合非完美型 & EU595409 \\
\hline
\end{tabular}

根据 Weber ${ }^{[29]}$ 提出的微卫星评价标准, 对 78 个 微卫星进行划分, 其中完美型(perfect)共 50 个, 占 64\%; 非完美型(imperfect)3 个，占 3.8\%; 混合完美 型(compound perfect)6 个, 占 $7.7 \%$; 混合非完美型 (compound imperfect) 19 个, 占 $24.5 \%$ 。其中完美型 的微卫星占大多数。

\section{3 多态性微卫星引物的设计及篮选结果}

选取 24 个微卫星 DNA 序列设计引物。经过初
步篎选，有 20 对能够扩增出产物，但只有 15 对适合 微卫星多态性分析。其中, 引物 3 对 20 个中国龙虾 个体微卫星位点检测的聚丙烯酰胺凝胶电泳见图 2。

\section{4 微卫星位点的多态性分析}

利用所获得微卫星引物对中国龙虾的一个群体 (20 个个体)进行遗传分析。对每一个微卫星位点的 等位基因数目(其中纯合个体的等位基因以出现两 次计算)进行统计。根据各个微卫星位点的等位基因

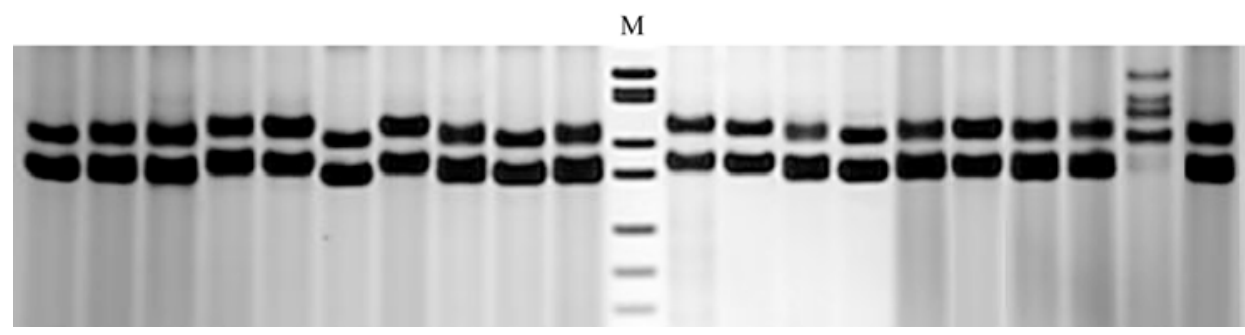

图 2 引物 3 在 20 个中国龙虾个体中的扩增结果 M: pBR322/Msp I DNA Markers。 
频率, 分别计算它们的期望杂合度 $(H e)$ 、观测杂合度 $(H o)$ 、多态信息含量 $(P I C)$ 和 Hardy-Weinberg 平衡指 数(HWE P)(表 2)。

从表 2 中可以看到，在中国龙虾 20 个样本的 15 个微卫星位点上, 等位基因的数目从 3 到 12 不等, 等位基因的大小分布在 78 425 bp 之间，基本符合 引物设计时的理论产物长度。这些微卫星位点的期 望杂合度的范围从 0.48 到 0.87 之间, 平均值为 0.71 , 表明它们都有较高的杂合度。15 个微卫星位点的 PIC 值从 0.44 到 0.84 , 平均值为 0.64 , 表明篮选到 的这些微卫星标记位点在中国龙虾中均具有较高的 信息含量, 可以应用于中国龙虾的分子遗传标记分 析和遗传图谱的构建。Hardy-Weinberg 平衡指数从 0.0000 到 0.6257 ，除位点 2 和 3 外的 13 个微卫星位 点均偏离 Hardy-Weinberg 平衡 $(P<0.05)$ 。
3 讨 论

\section{1 引物设计时微卫星序列的选取}

在微卫星标记的篮选过程中, 微卫星序列的篮 选是非常重要和关键的。根据 Weber 提出的分类标 准 ${ }^{[29]}$, 微卫星的核心序列有各种各样的类型: 完美 型(perfect), 非完美型(imperfect)和混合型(compound), 又可以分为混合完美型(compound perfect)和混合非 完美型(compound imperfect)。但关于使用哪种类型 核心序列的微卫星来设计微卫星引物才能获得具有 较高多态性的微卫星标记, 一直以来众说纷纭。在 本研究中, 主要选取了两碱基和三碱基为基本重复 单位的微卫星序列进行微卫星引物的设计, 且多为 compound imperfect 类型。最终结果表明, 使用自行 设计的微卫星引物进行 PCR 扩增, 最后的产物

表 2 中国龙虾 15 个微卫星位点的等位基因数、观测杂合度、期望杂合度、多态信息含量及 Hardy-Weinberg 平衡指数

\begin{tabular}{|c|c|c|c|c|c|c|c|c|}
\hline 微卫星标记 & $\begin{array}{l}\text { 引物序列 } \\
\left(5^{\prime} \rightarrow 3^{\prime}\right)\end{array}$ & $\begin{array}{l}\text { 复性 } \\
\text { 温度 } \\
\left({ }^{\circ} \mathrm{C}\right)\end{array}$ & 样本数 & $\begin{array}{c}\text { 等位基因个数 } \\
\text { (bp) }\end{array}$ & $\begin{array}{c}\text { 期望杂合度 } \\
(\mathrm{He})\end{array}$ & $\begin{array}{c}\text { 度 观测杂合度 } \\
(\mathrm{Ho})\end{array}$ & $\begin{array}{l}\text { 多态信息含量 } \\
(P I C)\end{array}$ & $\begin{array}{l}\text { Hardy-Weinberg } \\
\text { 平衡指数 }(P)\end{array}$ \\
\hline 引物 1 & $\begin{array}{l}\text { L: TTTATGGATGGGGCTGTT } \\
\text { R: CCTTGCTCTTATTCACATTTAC }\end{array}$ & 52 & 20 & $3(228 \sim 425)$ & 0.5949 & 1.0000 & 0.4918 & 0.0000 \\
\hline 引物 2 & $\begin{array}{l}\text { L: ACATGAATCAGCCACCAG } \\
\text { R: GGAAAGAGGGGCAAGTAT }\end{array}$ & 52 & 20 & $3(91 \sim 168)$ & 0.5603 & 0.9500 & 0.4422 & 0.6257 \\
\hline 引物 3 & $\begin{array}{l}\text { L: GAAGGAACGGATGTTAGA } \\
\text { R: AAAGGCATTCATTTAGGC }\end{array}$ & 54 & 20 & $12(104 \sim 145)$ & 0.8692 & 1.0000 & 0.8400 & 0.0539 \\
\hline 引物 5 & $\begin{array}{l}\text { L: TGGCAAGCTGGTGTCATG } \\
\text { R: TAGCCTCTGTATAAACATTTGG }\end{array}$ & 52 & 20 & $4(101 \sim 128)$ & 0.6769 & 1.0000 & 0.5958 & 0.0001 \\
\hline 引物 6 & $\begin{array}{l}\text { L: CACGGGATGATGGTATCGG } \\
\text { R: GGAGGAAGACCACGGAAA }\end{array}$ & 52 & 20 & $6(118 \sim 152)$ & 0.8385 & 1.0000 & 0.7914 & 0.0315 \\
\hline 引物 7 & $\begin{array}{l}\text { L: GGGAGGAAGACCACGGAAAC } \\
\text { R: GCGGCTCTGCTATTATGATTGT }\end{array}$ & 53 & 20 & $6(272 \sim 421)$ & 0.8487 & 1.0000 & 0.8033 & 0.0356 \\
\hline 引物 8 & $\begin{array}{l}\text { L: GCTGCCAGATGTTTCCAT } \\
\text { R: CAGGCTTTACACTTTATGCTTC }\end{array}$ & 54 & 20 & $4(78 \sim 125)$ & 0.6513 & 1.0000 & 0.5729 & 0.0000 \\
\hline 引物 11 & $\begin{array}{l}\text { L: TGGTCGATAGAATGGGTAC } \\
\text { R: TCACATCATCACGGGAGA }\end{array}$ & 54 & 20 & $4(98 \sim 126)$ & 0.7667 & 1.0000 & 0.7003 & 0.0061 \\
\hline 引物 14 & $\begin{array}{l}\text { L: CCGCATACGTCAAAGCAA } \\
\text { R: CGAGAAAGGAAGGGAAGAAA }\end{array}$ & 53 & 20 & $3(290 \sim 405)$ & 0.4810 & 1.0000 & 0.5478 & 0.0002 \\
\hline 引物 16 & $\begin{array}{l}\text { L: TTCTTGGTGTCAATGGGAGG } \\
\text { R: GTTGAGCCCAAACCTCCC }\end{array}$ & 52 & 20 & $4(155 \sim 185)$ & 0.7282 & 1.0000 & 0.6569 & 0.0016 \\
\hline 引物 18 & $\begin{array}{l}\text { L: TGTGCTGGAGGAGGATGT } \\
\text { R: CACCTTACCTTAGCCTCTGT }\end{array}$ & 52 & 20 & $4(105 \sim 123)$ & 0.7283 & 1.0000 & 0.6569 & 0.0012 \\
\hline 引物 19 & $\begin{array}{l}\text { L: ACGGGATGATGGTATCGG } \\
\text { R: GGAGGAAGACCACGGAAA }\end{array}$ & 52 & 20 & $4(322 \sim 370)$ & 0.7462 & 1.0000 & 0.6774 & 0.0028 \\
\hline 引物 20 & $\begin{array}{l}\text { L: GAAGAACGGATGTTAGA } \\
\text { R: AAAGGCATTCATTTAGGC }\end{array}$ & 53 & 20 & $3(125 \sim 180)$ & 0.6295 & 1.0000 & 0.5392 & 0.0000 \\
\hline 引物 22 & $\begin{array}{l}\text { L: AGATACCAGGCGTTTCCC } \\
\text { R: CAGAATGAATCTACGGTGACTA }\end{array}$ & 52 & 20 & $4(273 \sim 318)$ & 0.7462 & 1.0000 & 0.6774 & 0.0023 \\
\hline 引物 23 & $\begin{array}{l}\text { L: GCTGCCAGATGTTTCCAT } \\
\text { R: CAGGCTTTACACTTTATGCTTC }\end{array}$ & 52 & 20 & $4(186 \sim 224)$ & 0.7052 & 1.0000 & 0.6298 & 0.0002 \\
\hline
\end{tabular}


大部分能显示中国龙虾基因组 DNA 微卫星序列的 多态性, 多态性比例较高(约为 $67 \%$ )。

\section{2 中国龙虾的遗传多样性}

遗传杂合度 $(H)$ 又称基因多样度, 可反映各群体 在多个位点上的遗传变异。群体 $H$ 低, 表明该群体 的遗传一致性高, 群体的遗传变异少, 群体遗传多 样性低。在本研究中有些位点在中国龙虾中的杂合 度为 0 , 是因为这些引物没有表现出多态性, 在 PCR 扩增产物中只有一条带(也就是纯合子, 示单态性)。 引物间的杂合度差异比较大, 杂合度最高为 0.87 (位 点 3 ), 最低为 0.48 (位点 14 ), 这说明用少量的遗传标 记进行群体杂合度的检测结果不一定能够代表群体 的真实情况。本研究中运用了 15 个微卫星标记进行 检测, 可以在一定的程度上反映中国龙虾群体的情 况。这些微卫星位点的期望杂合度范围为 0.48 到 0.87 , 平均值为 0.71 , 表明中国龙虾基因组微卫星具有 较高的杂合度, 中国龙虾具有较高的遗传多样性。

多态信息含量 $(P I C)$ 是指一个后代所获得的某 个等位基因标记来自于它亲代的同一个等位标记的 可能性大小, 平均 PIC 是衡量等位基因片段多态性 的理想指标。当 $P I C$ 大于 0.5 时, 为高度多态位点; $0.25<P I C<0.5$ 时为中度多态位点; $P I C$ 小于 0.25 时为 低度多态位点 ${ }^{[30]}$ 。在本研究中, 15 个微卫星位点的 $P I C$ 值从 0.44 到 0.84 , 平均值为 0.60 , 说明这些微卫 星位点在中国龙虾基因组中包含丰富的遗传信息, 为高度多态位点, 适宜用于中国龙虾的各种分子标记 及遗传学分析和应用, 也表明了中国龙虾具有较高的 遗传多样性, 与遗传杂合度 $(H)$ 的分析结果相符合。

对中国龙虾 20 个样本的 15 个微卫星位点的 HWE 分析发现, 只有位点 2 和 3 处于平衡状态 $(P>0.05)$, 其 余 13 个微卫星位点均偏离 Hardy-Weinberg 平衡 $(P<0.05)$, 可能是现阶段中国龙虾资源已经受到人为 干扰等因素使其种群结构受到影响而产生。

一个群体内只有遗传多样性高或遗传变异丰富 时, 对环境变化的适应能力才较强, 才能比较容易 扩展其分布范围和拓展新的环境。虽然本研究表明 中国龙虾具有较高的遗传多样性, 但目前我国的龙 虾资源现状不容乐观。随着我国对海洋资源的进一 步开发和利用, 特别是近年来海水养殖行业的兴起, 我国的海洋环境正在不断的恶化，我国的龙虾资源 也面临着严重的威胁。这些都提示我们要采取科学的
措施最大限度地来保护和维持我国龙虾的自然资源和 遗传多样性，以保证可持续开发利用海洋渔业资源。

\section{参考文献(References):}

[1] 曾庆国, 陈艺燕. 微卫星位点篮选方法综述. 西北植物 学报, 2005, 24(4): 368-372.

[2] Wang Z, Weber JL, Zhong G, Tanksley SD. Survey of plant short tandem DNA repeats. Theor Appl Genet, 1994, 88(1): 1-6.

[3] Miyao A, Zhong HS, Monna L, Yano M, Yamamoto K, Havukkala I, Minobe Y, Sasaki T. Characterization and genetic mapping of simple sequence repeats in the rice genome. DNA Res, 1996, 3(4): 233-238.

[4] Norris AT, Bradley DG, Cunningham EP. Microsatellite genetic variation between and within farmed and wild Atlantic salmon (Salmo salar) populations. Aquaculture, 1999, 180(3-4): 247-264.

[5] 刘丽, 刘楚吾, 郭昱嵪, 董秋芬, 徐田军. 青石斑鱼微 卫星 DNA 标记的篮选及群体遗传多样性分析. 中国水 产科学, 2008(1): 22-29.

[6] 郭昱嵩, 王中铎, 刘楚吾, 刘筠. 勒氏笛鲷微卫星位点 的篮选及特征分析. 遗传, 2007, 29(3): 355-359.

[7] Bierne N, Launey S, Naciri-Graven Y, Bonhomme F. Early effect of inbreeding as revealed by microsatellite analyses on Ostrea edulis larvae. Genetics, 1998, 148(4): 1893-1906.

[8] 董秋芬, 刘楚吾, 郭昱嵩, 刘丽, 吴勇. 9 种石斑鱼遗传 多样性和系统发生关系的微卫星分析. 遗传, 2007, 29(7): 837-843.

[9] Kim HS, Lee BL, Woo DK, Bae SI, Kim WH. Assessment of markers for the identification of microsatellite instability phenotype in gastric neoplasms. Cancer letters, 2001, 164(1): 61-68.

[10] Bessert ML, Orti G. Microsatellite loci for paternity analysis in the fathead minnow, Pimephales promelas (Teleostei: $\mathrm{Cy}-$ prinidae). Mol Ecol Notes, 2003, 3(4): 532-534.

[11] Stockburger EM, Green RD, Wood WO, Holm T, Macneil MD, Schafer DW. Determination of the stringency of DNA microsatellite marker genotypes for use in individual animal identification. Anim Genet, 2000, 53: 345-348.

[12] 纪成林. 我国沿海经济甲壳动物之一——福建沿海的中 国龙虾. 水产科技情报, 1976, (1): 13-14.

[13] 刘瑞玉. 我国黄、东海虾类动物地理学研究. 海洋与湖 沼, 1963, 5(3): 230-244.

[14] Talbot P, Summers RG. The structure of sperm from Panulirus, the spiny lobster, with special regard to the acrosome. J Ultrastruct Res, 1978, 64(3): 341-351. 
[15] 姜永华, 颜素芬. 中国龙虾卵子发生及卵黄发生的超微 结构观察. 中国水产科学, 2009, 16(5): 697-704.

[16] 椎野季雄. 水产无脊椎动物学. 东京: 培风馆, 1969: 335.

[17] Phillips BF, Brown PA, Rimmer DW, Reid DD. Distribution and dispersal of the phyllosoma larvae of the western rock lobster, Panulirus cygnus, in the south-eastern Indian Ocean. Aust J Mar Freshwat Res, 1979, 30(6): 773-783.

[18] Rimmer DW, Phillips BF. Diurnal migration and vertical distribution of phyllosoma larvae of the western rock lobster Panulirus cygnus. Mar Biol, 1979, 54(2): 109-124.

[19] Rimmer DW. Spatial and temporal distribution of early stage phyllosoma of western rock lobster Panulirus cygnus. Aust J Mar Freshwat Res, 1980, 31(4): 485-497.

[20] 姜永华, 颜素芬. $\mathrm{pH}$ 值对中国龙虾消化酶活力的影响. 动物学报, 2008, 54(002): 317-322.

[21] Inoue M, Nonaka M. Notes on the cultured larvae of the Japanese spiny lobster, Panulirus japonicus (V. Siebold). Bull Jap Soc Sci Fish, 1963, 29(3): 211-218.

[22] Garcia-Rodriguez FJ, Perez-Enriquez R. Genetic differentiation of the California spiny lobster Panulirus interruptus (Randall, 1840) along the west coast of the Baja California Peninsula, Mexico. Mar Biol, 2006, 148(3): 621-629.

[23] Chow S, Suzuki N, Imai H, Yoshimura T. Molecular species identification of spiny lobster phyllosoma larvae of the genus Panulirus from the northwestern Pacific. Mar Biotechnol, 2006, 8(3): 260-267.

[24] Diniz FM, Maclean N, Ogawa M, Paterson I G, Bentzen P. Microsatellites in the overexploited spiny lobster, Рanulirus argus: Isolation, characterization of loci and potential for intraspecific variability studies. Conserv Genet, 2005, 6(4): 637-641.

[25] Hodgins-Davis A, Roberts S, Cowan DF. Characterization of SSRs from the American lobster, Homarus americanus. Mol Ecol Notes, 2007, 7(2): 330-332.

[26] Groth DM, Lim F, de Lestang SN, Beale N, Melville-Smith R. Characterization of polymorphic microsatellite loci in the western rock lobster (Panulirus cygnus). Conserv Genet Resour, 2009, 1(1): 163-166.

[27] 卢圣栋. 现代分子生物学实验技术. 第二版. 北京: 中 国协和医科大学出版社, 1999, 61-69.

[28] Raymond M, Rousset F. GENEPOP (version 1.2): population genetics software for exact tests and ecumenism. $J$ Hered, 1995, 86: 248-249.

[29] Weber JL. Informativeness of human (dC-dA) n. (dG-dT) n polymorphisms. Genomics, 1990, 7(4): 524-530.

[30] Botstein D, White RL, Skolnick M, Davis RW. Construction of a genetic linkage map in man using restriction fragment length polymorphisms. Am J Hum Genet, 1980, 32(3): 314-331. 